Ana Carolina Bernardes TERZIAN ${ }^{1}$

Debora Aparecida Pires de Campos ZUCCARI ${ }^{2}$ Rodrigo Storti PEREIRA ${ }^{2}$ Marcília Viana PAVAM ${ }^{2}$ Camila Montanari RUIZ² Felipe Augusto Ruiz SUEIRO² Joanna COELHO

Correspondência para: ANA CAROLINA BERNARDES TERZIAN Av. Juscelino K. Oliveira, 1220 - casa 131 rua 02 - Jd. Panorama

15091-450-São José do Rio Preto -SP anacarolinaterzian@gmail.com

Recebido para publicação: 18/05/2005 Aprovado para publicação: 07/02/2007

\title{
Avaliação da caspase-3 e Ki-67 como marcadores prognósticos nas neoplasias mamárias em cadelas
}

\author{
1 - Laboratório de Pesquisa em Virologia do Departamento de Doenças \\ Infecciosas e Parasitárias da Faculdade de Medicina de São José do Rio \\ Preto, São José do Rio Preto - SP \\ 2 - Centro Universitário de Rio Preto, São José do Rio Preto - SP \\ ${ }^{+}$Graduanda em Medicina Veterinária do Centro Universitário de Rio Preto, \\ São José do Rio Preto - SP (in memorian)
}

\section{Resumo}

A apoptose e a proliferação celular possuem uma participação importante na tumorigênese, determinando o crescimento tumoral e consequentemente sua agressividade. O presente estudo teve como objetivo avaliar a ocorrência da apoptose associada a proliferação celular em neoplasias mamárias em cadelas e a elas a evolução clínica do paciente. Setenta animais foram submetidos à exérese cirúrgica do tumor, sendo este submetido ao diagnóstico histopatológico e marcação imuno-histoquímica para caspase-3 e Ki-67. Estes marcadores de apoptose e proliferação celular demonstraram grande expressão nas neoplasias malignas, principalmente nos carcinomas, considerado o mais maligno dos tumores. Estes resultados corroboram os dados da literatura e contribuem para um prognóstico tumoral criterioso que complementa a classificação tumoral existente proporcionando uma melhor e maior sobrevida devido a uma adequação do procedimento terapêutico de cada paciente.

\section{Introdução}

A glândula mamária é o local mais comumente afetado por neoplasias em fêmeas caninas sendo que estas representam $50 \%$ de todos os tumores nesta espécie ${ }^{1,2,3,4,5,6,7,8}$. Além disso, atualmente surgiu um especial interesse no estudo dos tumores das glândulas mamárias em cadelas devido à sua semelhança com o câncer de mama em mulheres ${ }^{3,9,10}$. Muitos estudos tentam esclarecer os mecanismos da carcinogênese e o avanço da biologia molecular e das técnicas de clonagem do DNA, levam a um melhor entendimento dos complexos eventos que ocorrem durante a transformação maligna dos tumores ${ }^{11}$, ao desenvolvimento de novas terapias contra o câncer ${ }^{12,13,14}$ para com isso melhorar o prognóstico do paciente ${ }^{11,15,16}$.

As alterações moleculares que são observadas nas células do paciente com câncer, começam a ser compreendidas e estão relacionadas com os mecanismos que regulam a divisão celular normal, a sobrevivência e a morte celular ${ }^{17,18}$. A morte celular programada, também chamada de apoptose, tem um papel importante na determinação do crescimento tumoral e sua agressividade $^{19}$. Sendo assim, a apoptose é considerada um mecanismo celular intrínseco e regulado, através do qual há um controle entre a produção de novas células e a capacidade individual das células em se autodestruírem ${ }^{20,21,22}$. Esse processo é de fundamental importância para o homem, pois a homeostase depende da habilidade do organismo em produzir novas células, e da habilidade individual destas se autodestruírem quando se tornam desnecessárias ou descoordenadas ${ }^{11,17,18}$.

O início do processo apoptótico é rigidamente controlado por numerosos sinais intracelulares e extracelulares capazes 
de induzir à morte celular programada ${ }^{13,22}$. Este processo celular envolve determinadas proteases, chamadas de caspases, que são ativadas pela clivagem proteolítica em resposta a sinais que induzem à apoptose. Essas proteases ativas clivam proteínas chaves nas células e as matam rápida $\mathrm{e}$ ordenadamente ${ }^{17,18}$. A regulação desse processo é tão complexa quanto a regulação do crescimento celular e acompanham um grupo de alterações bioquímicas e morfológicas características ${ }^{13}$, com alterações celulares $^{23}$.

A caspase-3 vem sendo foco de estudos relacionados ao mecanismo da apoptose, tornando-se peça-chave no entendimento dos mecanismos de morte celular, uma vez que é ativada em resposta a vários estímulos, como drogas quimioterápicas. Segundo O'Donavan et al. ${ }^{24}$, a marcação da caspase-3 pode ser usada no controle da resposta aos tratamentos quimioterápicos quanto a possível resistência da droga, onde a diminuição da expressão pode indicar um mecanismo importante de sobrevivência celular em pacientes com câncer de $\operatorname{mama}^{27,10}$.

Wu, Shao e Jiang ${ }^{26}$ mostraram que o índice de apoptose é um fator prognóstico independente, mas Gonzáles-Cámpora et al. ${ }^{19}$ demonstraram que a estimativa da apoptose é um fator de prognóstico independente, assim como os índices de proliferação celular ou a expressão da proteína bcl-2 (inibidor da apoptose) ou p53 (indutor da apoptose). Sendo assim, a mensuração da proliferação e da apoptose podem promover um prognóstico real do comportamento tumoral ${ }^{27}$.

Segundo Arends ${ }^{28}$, a utilização dos marcadores de apoptose é considerada muito importante para caracterizar biologicamente o tumor, pois este fenômeno possui um papel fundamental na tumorigênese $\mathrm{e}^{27}$.

O Ki-67 é uma proteína nãohistona que não é expressa em células na fase G0, mas que pode ser detectada nas fases ativas do ciclo celular (G1, S, G2 e mitose). Inúmeros estudos tentam mostrar a relação entre o número de células Ki-67 positivas e o bom ou mau prognóstico em relação ao câncer de mama ${ }^{29}$. Zuccari ${ }^{30}$ aponta o Ki-67 como um excelente marcador de proliferação celular no diagnóstico e prognóstico de carcinomas mamários e para a malignidade em tumores mamários em cadelas. Verificou-se que o Ki-67 está associado com a proliferação celular, estando a fração de células tumorais positivas ao Ki67 correlacionada com a evolução clínica da doença.

Dessa maneira, considerando-se a importância da apoptose e da proliferação celular no desenvolvimento das neoplasias e o desconhecimento da sua real atividade nas neoplasias mamárias caninas, o presente estudo teve como objetivo verificar estes fenômenos pela marcação imuno-histoquímica da caspase-3 e Ki-67 e sua correlação com o diagnóstico histopatológico e o prognóstico nas neoplasias mamárias.

\section{Material e Métodos}

Foram utilizados 70 (setenta) fêmeas caninas, portadoras de neoplasias mamárias atendidas junto ao Hospital Veterinário "Dr. Halim Atique" - UNIRP - câmpus II e submetidas à exérese cirúrgica tumoral no período de Agosto de 2003 a Julho de 2005. Os fragmentos tumorais foram fixados em formol 10\% tamponada por até 24 horas e incluídos em parafina, sendo submetidos a cortes histológicos de $5 \mathrm{~mm}$ de espessura. As lâminas foram submetidas à técnica de Hematoxilina e Eosina (HE) para o diagnóstico histopatológico e as técnicas de imuno-histoquímica para a detecção da apoptose e da proliferação celular pela expressão de, respectivamente, caspase- 3 e $\mathrm{Ki}-67$.

Após a exérese tumoral, os animais foram acompanhados a cada 6 meses por 18 meses, estimando-se o tempo de sobrevida e o tempo livre da doença (surgimento de metástases e/ou recidivas).

Positividade ao exame imunohistoquímico para a caspase-3 e Ki-67.

Após desparafinização, seguiu-se o 
bloqueio da peroxidase endógena com água oxigenada (10V) por 30 minutos (3 banhos de 10 minutos), seguida de consecutivas lavagens em água destilada (3 banhos). A seguir realizou-se a recuperação antigênica em panela a vapor (entre $95^{\circ}$ e $98^{\circ} \mathrm{C}$ ) com solução de tampão citrato $(\mathrm{pH} 6,0)$ por 35 minutos e estabilizou-se as lâminas. Após, realizaram-se banhos em água corrente, água destilada e solução de PBS, sucessivamente. Fez-se a incubação com o anticorpo primário, em câmara úmida ("over night"), a $4^{\circ} \mathrm{C}$, com o anticorpo anti-caspase-3 (clone JHM62, Novocastra $\left.{ }^{\circledR}\right)$ na diluição de 1:100 e com a anticorpo anti-Ki-67 (clone MB-1, Novocastra ${ }^{\circledR}$ ) na diluição de 1:100. Após estabilização das lâminas a temperatura ambiente e banhos com solução de PBS, as lâminas foram incubadas com anticorpo secundário (Solução A - kit Dako LSAB+, Peroxidase-Universal) por 1 hora à temperatura ambiente. Após banho com PBS, as lâminas foram conjugadas com o Complexo Strepto-avidina/Peroxidase (Solução B - kit Dako LSAB+, PeroxidaseUniversal) por 30 minutos. Após banho em PBS, houve a revelação com DAB $(20 \mu \mathrm{l}$ de cromogen $+1 \mathrm{ml}$ de Buffered, Dako ${ }^{\circledR}$ ) e contra-coloração com Hematoxilina de Harrys. Após desidratação e diafanização, as lâminas foram montadas em meio vermount.

Todas as fases de preparação das lâminas foram acompanhadas por um controle positivo definido no folder do anticorpo para o anticorpo testado e um controle negativo (sem anticorpo primário). A distribuição do marcador foi verificada de acordo com a graduação de expressão proposta por Allred ${ }^{31}$ no sistema de cruzes (Figura 1). De acordo com essa técnica são contados 10 campos com 1000 células e feita média classificando-se pelo número de células marcadas.

A análise estatística utilizou o Teste de Fisher, aplicado a um pequeno $\mathrm{n}^{\circ} \mathrm{de}$ amostras e a Regressão Logística Nominal, quando considerado apenas o diagnóstico. Essas análises avaliaram a influência de um conjunto de variáveis com uma variável dependente (recidivas, metástases, tempo livre da doença, e sobrevida). As análises foram baseadas no diagnóstico tumoral e na evolução clínica do paciente, usando a expressão da Caspase-3 e Ki-67; o intervalo livre da doença, recidiva e metástase (classificação TNM) e os registros de óbito (animal que veio a óbito após cirurgia), eutanasiado devido à neoplasia (a pedido do proprietário), ou vivo (animais que permaneceram vivos até 18 meses póscirurgia) também foram considerados.

\section{Resultados e Discussão}

Em relação à evolução da doença, a cada 6 meses pós-exérese tumoral, o estado de saúde geral das cadelas foi avaliado. Das cadelas reavaliadas, $62 \%$ não apresentaram recidiva ou metástase (avaliada através de exame radiográfico), sendo que $7 \%$ apresentaram recidivas (5 fêmeas); $22 \%$ vieram a óbito pelo tumor; $7 \%$ foram submetidas a eutanásia e 9\% vieram a óbito devido a outros problemas, sem relação com a neoplasia mamária.

O exame histopatológico demonstrou que os tumores de maior freqüência foram os carcinomas, com $65 \%$ dos casos. Destes, $41 \%$ eram tubulopapilíferos, $36 \%$ complexos, $17 \%$ sólidos e $6 \%$ anaplásicos, esquirrosos ou escamosos. Os tumores mistos malignos somaram $21 \%$ do total e foram agrupados conforme o tipo de metaplasia. Destes, 54\% apresentavam metaplasia cartilaginosa, 13\% metaplasia óssea e 33\% apresentavam os dois tipos de metaplasia.. As neoplasias benignas totalizaram $14 \%$ dos casos, e foram representadas pelas hiperplasias ductais $(60 \%)$ e pelos tumores mistos benignos (40\%).

O exame histopatológico quantificou a presença de mitoses sendo que, $81 \%$ dos cortes apresentaram baixo número de figuras mitóticas e $19 \%$ apresentaram mitoses em grau moderado.

Nos resultados imuno-histoquímicos a expressão da caspase-3 apresentou-se 
Tabela 1 - Expressão da Caspase-3 em relação ao diagnóstico histopatológico nas neoplasias mamárias de cadelas segundo a classificação de ALLRED etal. (1998)

\begin{tabular}{|c|c|c|c|c|c|c|}
\hline Sistema de Cruzes & Negativo & Focal & Até $25 \%$ & $25-50 \%$ & $>50 \%$ & (\%) \\
\hline $\begin{array}{l}\text { Histopatológico } \\
\text { (número de casos) }\end{array}$ & - & + & ++ & +++ & ++++ & Total Global \\
\hline Benigno & 1 & 2 & 3 & 4 & 4 & $20 \%$ \\
\hline Carcinoma & 2 & 5 & 4 & 13 & 15 & $55,7 \%$ \\
\hline Misto Maligno & 2 & 2 & 3 & 5 & 5 & $24,3 \%$ \\
\hline Total Global (\%) & $7,1 \%$ & $12,9 \%$ & $14,3 \%$ & $31,4 \%$ & $34,3 \%$ & $100 \%$ \\
\hline
\end{tabular}

Tabela 2- Expressão do Ki-67 em relação ao diagnóstico histopatológico nas neoplasias mamárias de cadelas, segundo classificação de ALLRED etal. (1998)

\begin{tabular}{lcccccc}
\hline Sistema de Cruzes & Negativo & Focal & Até $25 \%$ & $25-50 \%$ & $>50 \%$ & ( \%) \\
\cline { 1 - 5 } $\begin{array}{l}\text { Histopatológico } \\
\text { (número de casos) }\end{array}$ & - & + & ++ & +++ & ++++ & Total Global \\
\hline Benigno & 2 & 2 & 1 & 3 & 4 & $17,1 \%$ \\
Carcinoma & 3 & 4 & 5 & 12 & 18 & $60 \%$ \\
Misto Maligno & 0 & 2 & 3 & 4 & 7 & $22,9 \%$ \\
\hline Total Global (\%) & $7,1 \%$ & $11,4 \%$ & $12,9 \%$ & $27,2 \%$ & $41,4 \%$ & $100 \%$ \\
\hline
\end{tabular}

fortemente positiva $(++++)$ em $65,7 \%$ dos fragmentos tumorais e em $34,3 \%$ dos fragmentos estavam negativas ou ++ . Quanto à expressão do marcador Ki-67, esta foi fortemente positiva em $68,6 \%$ das neoplasias estudadas e em $31,4 \%$, negativa ou positiva em até $25 \%$ das células (Tabelas 1 e 2$)$.

Pelo Teste de Fisher observou-se que houve uma correlação positiva entre o prognóstico ruim e o índice de apoptose $(\mathrm{P}=0,005)$ nos animais avaliados (Figura 2A). Não houve correlação com os carcinomas.

Pela Regressão Logística Nominal, houve evidência de associação entre a expressão do Ki-67 e o diagnóstico de carcinoma $(\mathrm{P}=0,016)$ quando consideramos apenas expressão positiva e negativa. Este resultado confirma a literatura existente que considera o Ki-67 um bom marcador para carcinomas e bom marcador prognóstico ${ }^{29,32,33}$ (Figura 2B).

De acordo com os autores Alberts ${ }^{17,18}$ e London e Vail $^{11}$ a apoptose pode ser considerada um marcador prognóstico favorável para indivíduos com neoplasia mamária uma vez que a homeostase que controla a morte celular programada estabiliza o crescimento e impede evolução tumoral. Por outro lado, durante o crescimento tumoral há um equilíbrio entre proliferação celular e apoptose $e^{34}$. Dessa maneira, se a presença de um grande número de marcadores apoptóticos possuir grande correlação com a proliferação celular, o indivíduo apresentará um prognóstico menos favorável ${ }^{27}$. Por isso, pode se deduzir que indivíduos com grande número de células marcadas pela apoptose possuem uma sobrevida menor ${ }^{19}$.

Também conforme estudos realizados por Wong et al. ${ }^{35}$; Mommers et al. ${ }^{36}$; Zhao et al. ${ }^{37}$; Parton et al. ${ }^{38}$, a correlação entre índice apoptótico e proliferação celular se mostrou bastante positiva, principalmente em carcinomas mamários, indicando que a apoptose está diretamente relacionada à taxa de proliferação tumoral na tentativa de controlar seu crescimento. Quanto à caspase3, o alto número de células apoptóticas está relacionado a um prognóstico não favorável. Considera-se que, se o índice de 

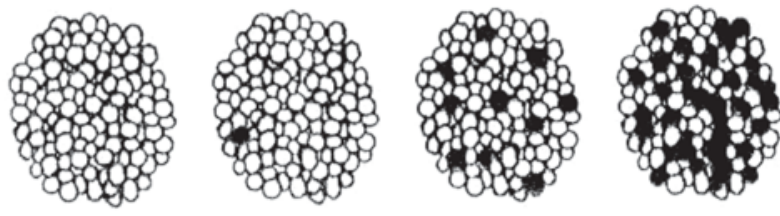

Negativo

(-)
Focal

$(+)$ até $25 \%$

$(++)$

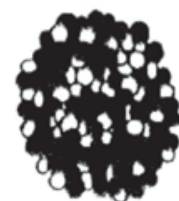

mais de $\mathbf{5 0 \%}$ $(++++)$

Figura 1 - Graduação de expressão no Sistema de Cruzes para avaliação imuno-histoquímica do marcador nas neoplasias mamárias

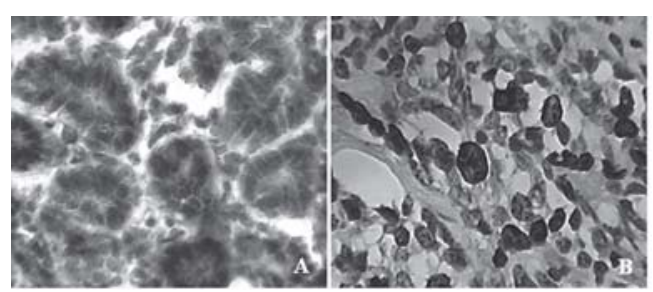

Figura 2 - Fotomicrografia da expressão imuno-histoquímica de $+++(25$ a $50 \%$ células $)$ para o anticorpo anti-caspase 3 (A) em Carcinoma Ductal e do anticorpo anti-Ki-67 (B) em Tumor Misto Maligno em cadelas. A marcação se caracteriza por ser citoplasmática em (A, 10X) e nuclear em $(B, 40 X)$

apoptose apresenta-se alto, o tumor cresce muito lentamente e se este estiver baixo, o tumor cresce rapidamente ${ }^{22}$. Isto é explicado porque durante o crescimento tumoral há um balanço entre a proliferação e a morte celular, onde o processo de apoptose está envolvido ${ }^{34}$. Sendo assim, o alto número de células apoptóticas juntamente com um alto índice de proliferação celular representam um prognóstico não favorável ${ }^{27}$.

De acordo com os resultados obtidos, e com Gonzáles-Cámpora et al. ${ }^{19} \mathrm{e}$

\section{Evaluation of caspase-3 and Ki-67 as a prognost mammary tumors \\ a prognostic markers in canine}

\section{Abstract}

Apoptosis, as a cellular event, has an important participation in tumorigenesis, determining the tumoral growth and aggressiveness. The present study had as objective to evaluate the occurrence of apoptosis, associated cellular proliferation, in canine mammary neoplasias and the patient clinical evolution. Seventy dogs had been
De Jong et al. ${ }^{27}$, houve correlação estatística de um alto número de células marcadas pela apoptose com a alta proliferação celular. Considerando-se que a intensa expressão do Ki-67 está relacionada à proliferação celular, que é uma característica de malignidade, os resultados comprovam a literatura.

\section{Conclusão}

Sendo assim, os resultados deste trabalho demonstram que estes marcadores podem ser utilizados como prognósticos nas neoplasias mamárias em cadelas, complementando a classificação tumoral existente e proporcionando assim, uma melhor e maior sobrevida ao paciente devido a possibilidade de individualização da terapia, principalmente nos casos de carcinomas mamários.

\section{Agradecimentos}

À Agência Fapesp pelo financiamento deste projeto (Proc. $\mathrm{n}^{\circ}$ 02/05336-2).

Key-words:

Mammary tumors.

Bitch.

Immunohistochemical.

Apoptosis.

Cellular proliferation 
submitted to surgical excision of the tumor fragment and they were submitted to the histopathologic diagnosis and imunohistochemistry procedure to caspase- 3 and the $\mathrm{Ki}-67$. This apoptosis and cellular proliferation markers demonstrated great expression in malignant neoplasias especially carcinoma, considered the most malignant of the tumors. These results confirm consulted literature contributing for a criterious tumoral prognostic complementing the tumoral classification, providing a greater and better supervened due to adequacy of therapeutic procedure of each patient.

\section{Referências}

1 DORN, C. R. et al. Survey of animal neoplasms in Alameda and Contra Costa Counties, California II. Cancer morbidity in dogs and cats from Alameda County. Journal of National Cancer Institute, v. 40, v. 2, p. 307-318, 1968.

2 MOULTON, J. E. Tumors of the mammary gland. In MOULTON, J. E. Tumors in domestic animals. 3. ed. London: University of California, 1990. p. 518-552.

3 PELETEIRO, M. C. Tumores mamários na cadela e na gata. Revista Portuguesa de Ciências Veterinárias, v. 89, n. 509, p. 10-29, 1994.

4 MACEWEN, E. G., WITHROW, J. S. Tumors of the mammary gland. In: . Small animal clinical oncology. Philadelphia: W.B. Saunders, 1996. p.356-72.

$5 \mathrm{O}^{\prime}$ KEEFE, D. A. Tumores do sistema genital e glândulas mamárias. In: ETTINGER, S. J.; FELDMAN, E. C.; Tratado de medicina interna veterinária. 4. ed. São Paulo: Manole, 1997, v. 2, p. 2348-2350.

6 DALECK, C. R. ET AL. Aspectos clínico e cirúrgico do tumor mamário canino. Ciência Rural, Santa Maria, v. 28, n. 1, p. 95-100, 1998.

7 JOHNSION, S. D. Sistemas reprodutivos. In: SLATTER, D. Manual de cirurgia de pequenos animais. São Paulo: Manole, 1998. v. 2. p. 2575-2581.

8 FONSECA, C. S.; DALECK, C. R. Neoplasias mamárias em cadelas: influência hormonal e efeitos da ováriohisterectomia como terapia adjuvante. Ciência Rural, Santa Maria, v. 30, n. 4, p. 731-735, 2000. Disponível em: < http://www.ufsm.br/revista/resumos/rv304/ rv304 1121.html > Acesso em: 14 abr. 2001

9 GILBERTSON, S. R. et al. Canine mammary epithelial neoplasms: biologic implications of morphologic characteristics assessed in 232 dogs. Veterinary Pathology, v. 20, p. 127-142, 1983.

10 NERURKAR, V. R. ET AL. Comparative pathology of canine mammary tumors. Journal of Comparative Pathology, v. 101, n. 4, p. 389-397, 1989.

11 LONDON, C. A., VAIL, D. M. Tumor biology. In: WITHOW, S. J.:MACEWEN, E. G. Small animal clinical oncology. 2. ed. Philadelphia: Saunders, 1996. p. 16 31.
12 HICKMAN, J. A. Apoptosis induced by anticancer drugs. Cancer Metastasis Review, v. 11, p. 121-139, 1992.

13 MILLER, D. M. O futuro da oncologia. In: BENNETT J. C.; PLUM, F.; Cecil: tratado de medicina interna. 20 ed. Rio de Janeiro: Guanabara Koogan, 1997. v. 1 e 2 p. 1183-1190.

14 BRYAN, G. T. Histórias naturais dos cânceres. In: LOVE, R. R. Manual de oncologia clínica. 6. ed. São Paulo: Springer-Verlog, 1999. p. 18-35

15 RICHARDSON, R. C. Spontaneous canine and feline neoplasm's as models for cancer in man. Kal Kan Forum v. 2, p. 89-94, 1983

16 MACEWEN, E. G. Spontaneous tumors in dogs and cats: models for the study of cancer biology and treatment. Cancer and Metastases Review, v. 9, n. 1, p. $125-136,1990$.

17 ALBERTS, B. et al. Controle do ciclo celular e morte celular. In: Fundamentos da biologia celular: uma introdução à biologia molecular da célula. Porto Alegre: Artmed, 1999. p. 585-607.

18 ALBERTS, B et al. Tecidos. In: Fundamentos da biologia celular: uma introdução a biologia molecular da célula. Porto Alegre: Artmed, 1999. p. 609-647.

19 GONZÁLES-CÁMPORA, R. et al. Apoptosis in Breast carcinoma. Pathology Research Practice, v. 196, n. 3 p. $167-174,2000$

20 CARSON, D. A.; RIBEIRO, J. M. Apoptosis and disease. Lancet, v. 341, p. 1251-1254, 1993

21 LICHTENFELS, P., BARRIOS, C. H., MOREIRA, L. F. Apoptose: morte celular programada. Acta Medica p. 298-311, 1999.

22 DUBIN, M.; STOPPANI, A. O. M. Muerte celula programada y apoptosis funcion de las mitocondrias. Medicina (Buenos Aires), v. 60, p. 375-386, 2000.

23 VAUX, D. L. Caspases and apoptosis-biology and terminology. Cell Death Differentiation, v. 6, p. 493 494,1999

24 O’DONOVAN, N. et al. Caspase 3 in breast cancer Clinical Cancer Research. v. 9, n. 2, p. 738-742, 2003.

25 DEVARAJAN, E. ET AL. Down-regulation of caspase 
3 in breast cancer: a possible mechanism for chemoresistance. Oncogene, v. 21, n. 57, p. 88438851. 2002.

26 WU, J. SHAO, Z., JIANG, M. In situ DNA labeling apoptosis in breast cancer as related to prognosis. Zhonghua Zhong Liu Za Zhi, v. 19, n. 2, p. 100-102, 1997.

27 DE JONG, J. S.; VAN DIEST, P. J.; BAAK, J. P .A. Number of apoptotic cells as a prognostic marker in invasive breast cancer. British Journal of Cancer, v. 82, n. 2, p. 368-373, 2000.

28 ARENDS, J. Effects of a combined thermochemotherapy on markers of apoptosis, differentiation and adhesion in the human mammary carcinoma MX-1. Annals of Anatomy, v. 182, p. 339347, 2000.

29 FITZGIBBONS P. L. et al. Prognostic factors in breast cancer. College of American Pathologists Consensus Statement 1999. Archives of Pathology \& Laboratory Medicine, v. 124, p. 966-978, 2000.

30 ZUCCARI, D. A. P. C. Estudo imunocitoquímico de marcadores diagnósticos e prognósticos em neoplasias mamárias caninas. 2001. 92 f. Tese (Doutorado em Clínica Médica Veterinária) - Faculdade de Ciências Agrárias e Veterinárias, Universidade Estadual Paulista, Jaboticabal, 2001.

31 ALLRED, C. D. et al. Prognostic and predictive factors in breast cancer by immunohistochemical analysis. Modern Pathology, v. 11, n. 2, p.155-168, 1998.

32 BOUZUBAR N. et al. Ki67 immunostaining in primary breast cancer: pathological and clinical associations. British Journal of Cancer, v. 59, n. 6, p. 943-947, 1989.

33 ZUCCARI, D. A. P. C.; SANTANA, A. E.; ROCHA, N. S. Correlação entre a citologia aspirativa por agulha fina e a histologia no diagnóstico de tumores mamários de cadelas. Brazilian Journal of Veterinary Research and Animal Science, v. 38, n. 1, p. 38-41, 2001.

34 SIMÃO, T. A et al. Detection and analysis of apoptosis in peripheral blood cells from breast cancer patients. Brazilian Journal of Medical and Biological Research, v. 32, n.. 4, p. 403-406. 1999.

35 WONG, S. C. C. et al. Differential expression of p16/p21/p27 and cyclin D1/D3, and their relationships to cell proliferation, apoptosis, and tumour progression in invasive ductal carcinoma of the breast. Journal of Pathology, v. 194, p. 35-42, 2001.

36 MOMMERS, E. C. M. et al. Balance of cell proliferation and apoptosis in breast carcinogenesis. Breast Cancer Research and Treatment, v. 58, p. 163 169, 1999.

$37 \mathrm{ZHAO}, \mathrm{H}$. et al. Immunohistochemical expression of uPA, PAI-1, cathepsin D and apoptotic cells in ductal carcinoma in situ of the breast. Breast Cancer, v. 9, p.
118-126, 2002

38 PARTON, M. et al. Coordinate expression of apoptosis-associated proteins in human breast cancer before and during chemotherapy. Clinical Cancer Research., v. 8, p. 2100-2108, 2002.

39 STONE, E. A. Neoplasia da glândula mamária. In: BIRCHARD, S. J.; SHERDING, R. G.; Manual Saunders: clínica de pequenos animais. São Paulo: Roca, 1998. p 234-237. 